\title{
A Computerized Suggested Electronic Tool for the Evaluation of the Attack's Technique of the Kumite Players in Karate
}

\author{
Ahmed Mahmoud Ibrahim*, EzzatAbdeltwapKorany**, Mohamed MohamedGalal***
}

\section{Introduction:}

$\mathrm{T}$ he new era of globalization and open sky world, pushes us to reevaluate and develop the way we are dealing with in all aspects. Training methods and the tools we are using have to be evaluated and developed as well. Since scientific development and researches are very important, creation and inventing are the best way to fulfill them. Physical training is a complete process and depends on scientific bases to serve different sides e.g. (skills, physical, and techniques) in addition to the modern tools and measuring devices to prepare the players for an international level of preparation.

\section{Research problem:}

Karate is one of the action games, where individuality, hard competition, and time requiring a highly prepared skillful player supported with good thinking and balance. Its international and local popularity pushed researchers to develop their researches especially in Kata and Kumite. Since the researcher noticed during his work as a trainer that the used tools for measuring and testing are so traditional and old fashion, creating a modern tool was essential to achieve the aim of developing the game. According to other researchers, computers and modern tools are essential to develop a contentiously developed game like Kumite.

*Professor of athletic training for karate, Department Combat training and individual sports Faculty of Physical Education for Boys Abu Qir,Alexandria University, Egypt.

**Professor of computer science, Head, Dept. of Information Technology, Institute of Graduate Studies and Research, Alexandria University, Egypt.

***Institute of Graduate Studies and Research, Alexandria University, Egypt.
Starting from this point, the researcher achieved a pre study to compare the naked eyed judging and the video-recorded review by the same judges. The results were completely different, and proved that a new tool to monitor the naked eyed judging is necessary. A computerized modern tool using modern device to measure and evaluate the attack's efficiency, with almost no mistakes, which reveals the curable negatives to save effort and expenses and increase the reliability of the scientific research. As many coaches and experts announced that the measuring and evaluation of the attack's effect is primitive although the high technology of karate training.

\section{Aim of the study:}

The study aims to propose a suggested training program to develop the efficiency of the attack of the karate players, by measuring the attack's efficiency using the suggested tool and the computer.

\section{Solutions:}

1. The suggested training program develops the efficiency of attack of karate players by measuring the attack's efficiency using the suggested tool and the computer.

2. Measuring the efficiency of attack by the electronic tool and the computer shows positive results in comparing with the traditional tools, and with no mistakes, which increases the efficiency of the training program. 


\section{Tool description:}

An electronic device consists of five units. It records the hits, which the player receives during the competition in the head and the upper part of his body. The recorded hits will be stored in a volatile memory until being displayed and statistically calculated.

\section{Tool components:}

\section{Electronic sensors}

2. Processing and recording unit (for the red player)

3. Processing and recording unit (for the blue player)

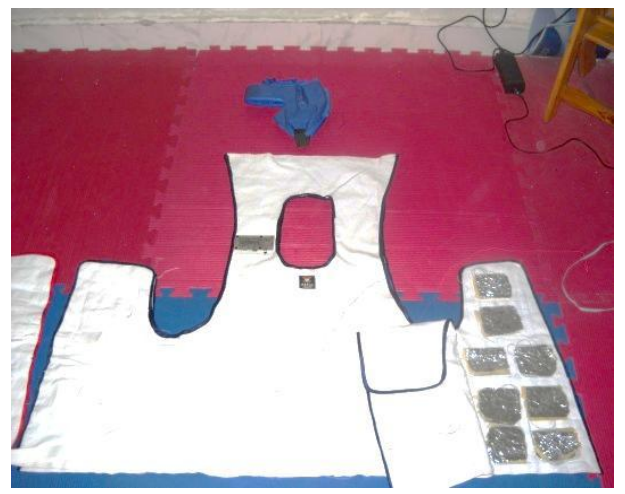

Fig. No. 1

Fig. No. 1 displays the main idea of the sensor

\section{Sensor structure:}

According to the nature of the environment, the sensor had to be suitable for using with human beings. Metal parts are not subject for discussion. That led to design a sensor made of soft components. The sensor has been made of sponge and two foil sheets. The sponge is the material, which folds the sensor and the isolation insert between the two foil sheets. While the two foil sheets are the switch terminals. Moreover, each terminal ends with a wire for electric connections. The sensor replies for forces between 100gm and a weight of a truck. And tested against heavy weight to ensure
4. The interface

\section{Software}

\section{Electronic sensors:}

Any affecting force caused by the competent will close normally open electronic switches, which are inserted between the tissues of the volts electric signal from terminal "A" to terminal "B" of the switch. The total number of sensors is 39 for each player, distributed as three sensors per line. Total lines are five lines, three each side of the body. The sensors are covering the chest area, the back area, the abdomen area, and the sides' areas. uniform. The external forces (hits) will pass 5 more sensors on the head, and three sensors on

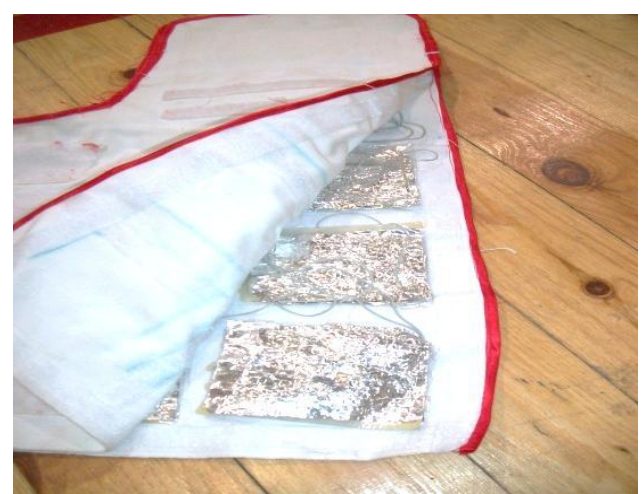

that it bears the nature of the competition. The sensor's dimension is $7 \mathrm{~cm} \times 5 \mathrm{~cm}$, and held in a proper pocket sawed to be a sensor pocket.

\section{Sensors' positions:}

The sensors are distributed on five raw forming five rings surrounding the upper part of the body. They cover the chest, the abdomen, the entire back, the two sides, forehead, and the head sides. Each raw contains three sensors on the front part of the body, and three other sensors on the back. In addition, the two sides are covered with six sensors. The total number of sensors is 39 sensors. 


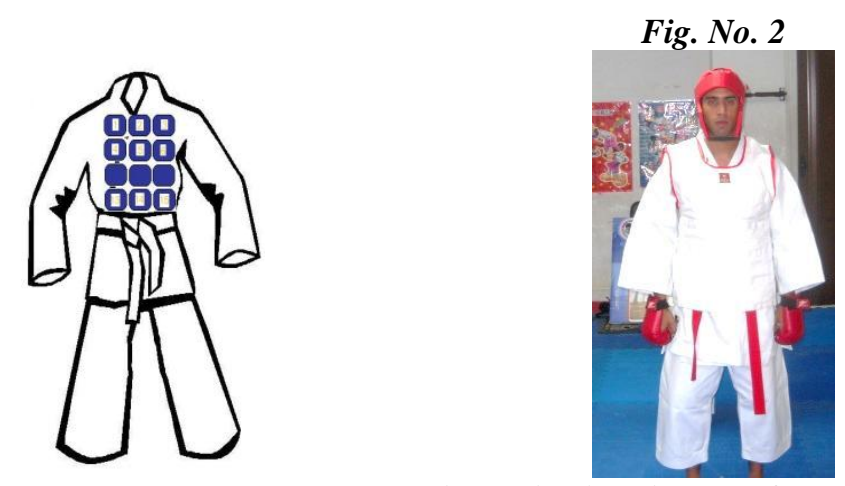

Fig. No. 2 shows the distribution of sensor inside the uniform

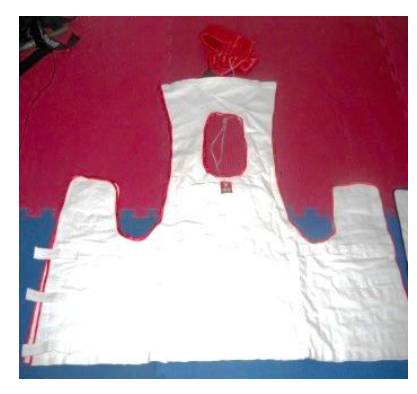

Fig. No. 3

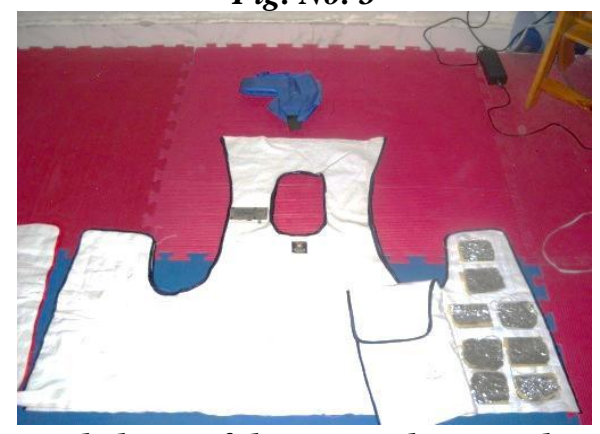

Fig. No. 3 shows real photos of the sensors between the uniform's layers

\section{Processing and recording unit:}

An electronic units its dimensions are $7 \mathrm{~cm} X$ $5 \mathrm{~cm} \mathrm{X} 0.5 \mathrm{~cm}$. contains necessary electronic components in addition to the CPU, which is the Programmable Intelligent Computer. The PIC is 16F877 microcontroller.

It is a chip with 40 pins 33 of them work as I/Ps or $\mathrm{O} / \mathrm{Ps}$ according to the program. The PIC works with $4 \mathrm{MHz}$ crystal, which allow it to perform one million process per second. It is equipped with a Master clear pin for a general reset function. It has its own memory modules. Part of the memory is EEPROM (Electrical erasable Programmable Read Only Memory) and the other part is RAM (Random Access Memory).

Both of these memories allow the chip to store digital data inside it without the need for an external memory module, as the capacity of the stored data is within its limit.

The type of data, which this PIC accepts is the digital data. Digital data are bits. Each bit contains 1 or 0 . The ones or zeros are not true digits, but they represent a case of true of false. Each eight bits form a byte. The byte is the unit of data, which digital electronics deals with.
Each byte has a combination of 256 different combinations of bits containing ones or zeros. The PIC like any other PC receives the data of this type and calculates its decimal values if needed.

Back to our tool, the sensors send its logic signals to the processing unit. The processing unit receives these data. Each pressed sensor sends the logic one to the processing unit, and sends zero if not pressed.

The sensors are connected to the PIC in a binary manner. In the binary data transmission, eight pins of the PIC can receive 256 different data based on the addressed data recognition. Which means, 255 digital sensors can be contained within eight $\mathrm{I} / \mathrm{P}$ pins of the PIC?

All sensors' data will be stored inside the memory module of the processing unit accompanied with its time and serial ordering. By connecting the processing unit to the $\mathrm{PC}$, the stored data is being transferred to the PC via the software program, where they will be processed.

The power supply of the processing unit is via 6 volts rechargeable battery runs the unit for about 4 hours. Each time the unit attached to the interface, the battery recharged. All the sensors 
are connected to the VCC with one of its terminals, while the other terminals pass the VCC to the PIC I/P pins when it is being pressed. The CPU stores the data in addition to the sensor's number (I.D.) and the time. The time is $0.01 \mathrm{Sec}$. and generated by the crystal time base.

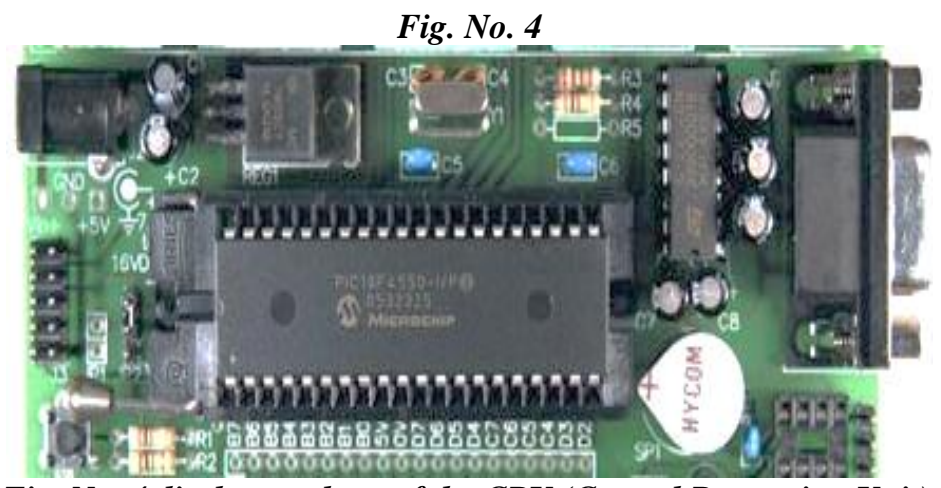

Fig. No. 4 displays a photo of the CPU (Central Processing Unit)

Fig. No. 5

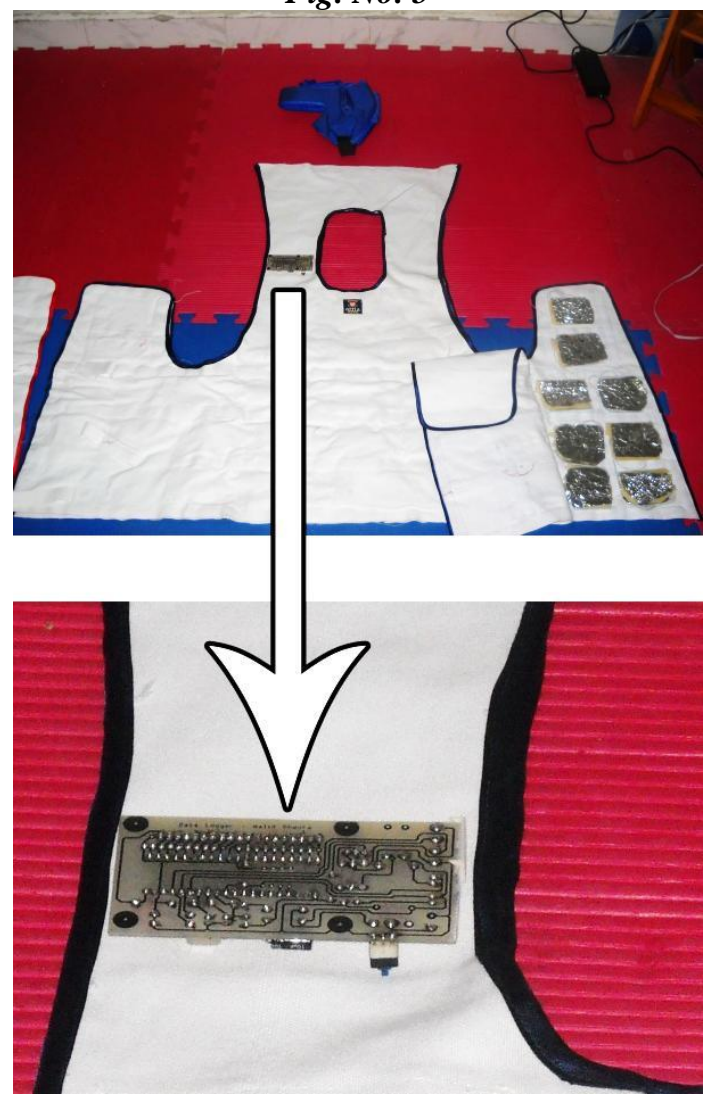

Fig. No. 5 displays the CPU position inside the uniform (On the shoulder)

\section{The interface:}

When attaching a PC to peripherals, the interfacing is necessary. The PC follows a certain routine to communicate with the outer world. That mean, a third part has to be between them to compete the peripheral with the PC. The interface is an interpreter between them. It is like a translator between two different languages. It solves a great connection's problem, since it is compatible with the PC from on side, and compatible with the peripheral on the other side. In addition, it has a third job in this project, since it recharges the battery of the processing unit each time it is connected to it. 


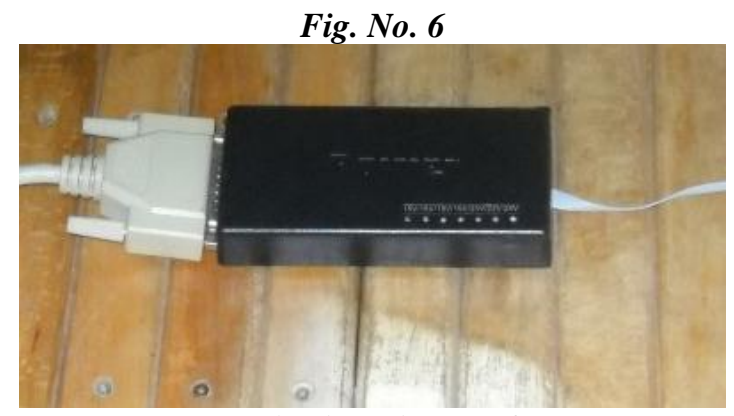

Fig. No. 6 displays the interface unit

\section{Software program:}

The manufacturer of that project designed the software program to read data from the processing unit and display it on the PC's monitor. The software is capable to communicate with the processing unit via printer's parallel port. It is also capable to display the result in tables showing the necessary data, which the researcher needs. In

Fig. No. 7

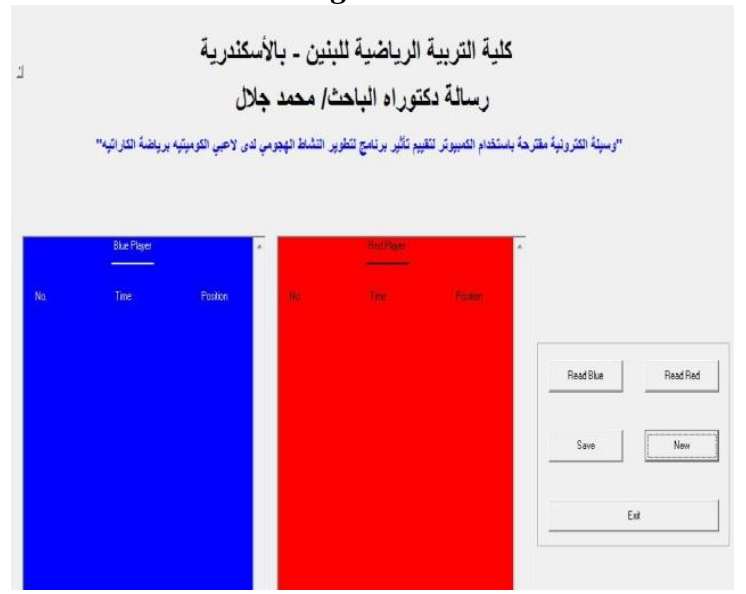

Fig. No. 7 displays the software interface with the control buttons

No
1
2
3
4
5
6

Time

00:00:23

00:01:06

00:00:23

00:01:06

00:00:23

00:01:06

\section{Position}

5

21

2

31

35

11 also provides the basic windows functions e.g. saving, opening, renaming, and printing. The output tables of the software are subject to be received by statistical and database programs. It is a kind of row data displayed in a fine ordering.

The software is written with visual basic. It is suitable to be used with Windows 95, windows vista, and Windows 7

Fig. No. 8

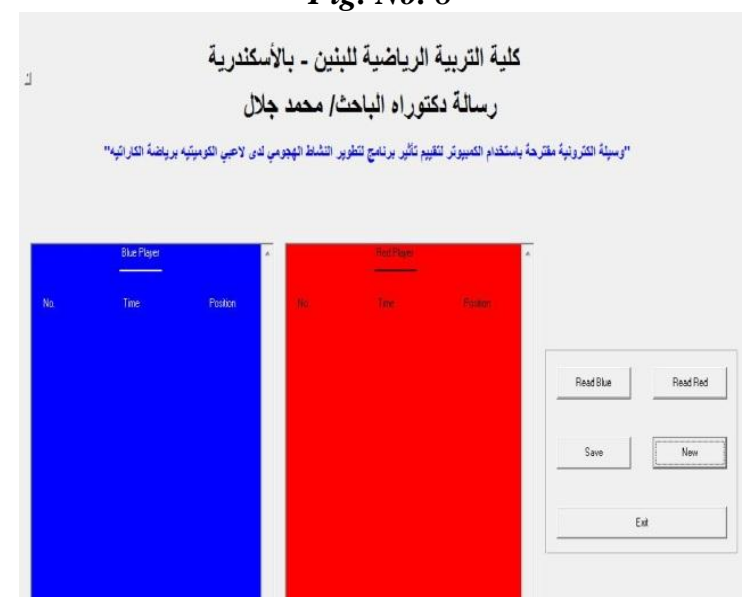

Fig. No. 8 shows recorded results of two players 


\section{Measuring steps:}

1. Each player wares his uniform and the head guard.

2. The researcher makes sure of the proper status of the device

3. Synchronizing resetting the Processing unit of each player

4. As the competition starts, each player tries to hit his competent and achieves the best score he can.

5. Each processing unit will record and store the hits of the other player .

6. The Processing unit will record the hit, the sensor's position, and the time

7. As the match ends, the processing units will be attached to the interface, and the software will be started to collect data from it

8. The software will display the result in the form of 1- 00:00:12 15

9. which means, hit No. 1 was at the first 12 seconds of the match at position No. 15

10. For collecting the other player's data, steps from step No.7

11. Save the files in a pre prepared folder to have good organized files

12. For a new measure, repeat from step N0. 1 till Step No. 10

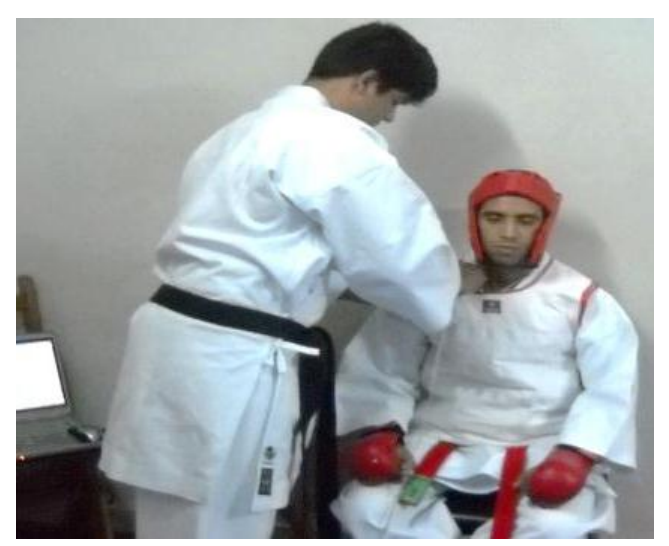

Main study:
The main study is designated for designing, preparing, producing, and evaluating the electronic tools.

\section{1- Designing of the electronic tool}

This stage started with a workshop attended by the researcher, the supervisors and the technician, who is responsible for the design and manufacturing of the electronic tools.

In this workshop, the technician interpreted the thoughts of the researcher and his supervisors into an electronic design considering the type of material, which is suitable for using with the human body during a competition and direct contact between two players.

\section{2- Probation of the electronic tool}

The main goal of this stage is to try the tool, test it, put it to work in all expected conditions, and to evaluate it.

The test of the tool had been done by using it by two high level players to figure out the obstacles of using the tool with humans, and to realize the required procedures, which are needed to be followed to get the best result of the tool and to avoid any disadvantages.

To achieve such a step, the tool received many random kicks with both hands and feet. Moreover, many actual matches have been done.

\section{The main goals of this stage:}

1. The suitability of the tool for the match.

2. Recognition of the difficulties of operation the tool.

3. The possibility data transfer from the tool to the PC.

4. Calculating the processing time of tool preparation \& data transfer.

5. Checking of tool's safety.

6. Verification of given result by using video 6 . analyzing.

7. Training the players to reduce the duration of putting on the uniform.

8. Checking of tool's reliability.

As these procedures have been done, the result came up positively. 
1. The electronic tool records precisely.

2. The tool does not respond to kicks out of its spots.

3. Data transfer needs 10 seconds for both players.

\section{References:}

1. Abdelrahman A. Abdelrahman, Alex. Uni: "A suggested electronicdevice for the determining of punches specificationfor the purpose of boxers selection for championships" Faculty of P.E. for men, PhD. 2006.

2. Ahmed M. Ibrahim, Alex. Uni: Scientific and applicable encyclopedia"Modern aspects and limits of evaluation styles and training's program planning of Karate". MonshaaElmaaref, Alex. 2012.

3. Ayman S. Abdelfatah,TantaUni: "Designing electronic gloves for boxing training". Faculty of P.E. for men, PhD. 2006

4. Ed Chi , Palo AltoUniv. : Martial arts land wireless blow ; Journal of BBC news technology, June 2004.
5. HayriErtan : Reliability and Validity testing of an archery chronometer, Journal of Sports Science and Medicine, volume 4,2005.

6. Kamal Abbas, Alex. Uni: "A computerized design for annual training program for swimming and Judo (A comparative study under 17 years old). Faculty of P.E. for men, 2006.

7. Roosen A. et al : A device to measure choice reaction time in karate., Journal of Sports Engineering, Volume 2, Number 1, June 1999.

8. Takahata Masami \&AoshimaDaigo\&Other: Extraction of players tactics in the karate match using ubiquitous sensing technology "Japan science and technology agency" Accession number: 04A0810905 Journal proceedings of the Annual conference on JSAI2007.

9. www. Science links Japan . com.

10. www. BBC/News/Technology. com. 